\title{
Frequency-comb-calibrated Doppler broadening thermometry
}

\author{
Davide Gatti, ${ }^{1,2}$ Andrew A. Mills, ${ }^{1}$ Maria Domenica De Vizia, ${ }^{3}$ Christian Mohr, ${ }^{1}$ Ingmar Hartl, ${ }^{4}$ Marco Marangoni, ${ }^{2}$ \\ Martin Fermann, ${ }^{1}$ and Livio Gianfrani ${ }^{3, *}$ \\ ${ }^{1}$ IMRA America, Inc., 1044 Woodridge Avenue, Ann Arbor, Michigan 48105, USA \\ ${ }^{2}$ Dipartimento di Fisica, Politecnico di Milano, Piazza Leonardo da Vinci 32, I-20133 Milano, Italy \\ ${ }^{3}$ Dipartimento di Matematica e Fisica, Seconda Università di Napoli, Via Vivaldi 43, I-81100 Caserta, Italy \\ ${ }^{4}$ Deutsches Elektronen-Synchrotron, Notkestrasse 85, D-22607 Hamburg, Germany
}

(Received 22 March 2013; published 17 July 2013)

\begin{abstract}
We describe an implementation of Doppler broadening thermometry based upon a room-temperature continuous-wave quantum cascade laser at $9.07 \mu \mathrm{m}$, coherently phase locked to a thulium optical frequency comb centered at $2 \mu \mathrm{m}$. The thermodynamic temperature is retrieved from the analysis of multiple-line absorption spectra of the $\mathrm{NH}_{3} v_{2}$ band, adopting semiclassical line-shape models and taking into full account the speed dependence of collisional broadening. We demonstrate that a precision of approximately five parts over $10^{5} \mathrm{can}$ be reached as a result of the analysis of only 90 absorption spectra, as acquired as a function of the gas pressure.

DOI: 10.1103/PhysRevA.88.012514

PACS number(s): 33.20.Ea, 42.62.Eh, 33.70.Jg, 42.62.Fi
\end{abstract}

\section{INTRODUCTION}

Doppler broadening thermometry (DBT) is a relatively new method for determining the absolute temperature of a gaseous system at thermodynamic equilibrium. Soon after its first implementation, this method appeared to be very promising, with the advantage of being conceptually simple, applicable to any gas at any temperature, in whatever spectral region [1]. It consists in retrieving the Doppler width from the highly accurate observation of the absorption profile corresponding to a given atomic or molecular line. Ammonia was the first thermometric substance on which DBT was tested, followed by carbon dioxide. In the former case a narrow $\mathrm{CO}_{2}$ laser, frequency stabilized on an $\mathrm{OsO}_{4}$ saturated absorption line, was used to probe a strong ${ }^{14} \mathrm{NH}_{3}$ line at $10.34 \mu \mathrm{m}$ [2]. In the subsequent implementation an extended-cavity diode laser (ECDL) at $2 \mu \mathrm{m}$ was used to interrogate a vibration-rotation transition of $\mathrm{CO}_{2}$ [3].

A highly linear, repeatable, and accurate frequency scale represents an indispensable prerequisite for a successful DBT experiment $[4,5]$. In this respect, an interesting approach was proposed by Castrillo et al. who employed the technique of offset-frequency locking between a pair of ECDLs at $1.38 \mu \mathrm{m}$ [6]. A more versatile scheme was implemented by Yamada et al. exploiting the technology of erbium-doped fiber-laser frequency comb synthesizers [7]. In their apparatus, an ECDL at $1.5 \mu \mathrm{m}$ was phase locked to the comb, and continuous frequency scans around a given ${ }^{13} \mathrm{C}_{2} \mathrm{H}_{2}$ line were performed by tuning the repetition rate, thus demonstrating a precision in Doppler-width retrieval of $1200 \mathrm{ppm}$. The present paper deals with the extension of this approach to the mid-infrared, taking the advantage of a new referencing scheme allowing a quantum cascade laser to be phase locked to a near-infrared frequency comb [8].

DBT not only requires a well-designed experiment where absorption profiles are measured with high fidelity, but also a very reliable physical modeling of the line shape, which may exhibit quite a strong departure from the usual Voigt profile. It

*Corresponding author: livio.gianfrani@unina2.it is well known that, besides the Doppler effect, other physical phenomena contribute to the line width and shape, including narrowing effects caused by velocity-changing collisions and speed-dependence of relaxation rates [9].

First-principles quantum-mechanical treatments of the shape of gas-phase spectra are so complex that even in the simplest case of a diatomic molecule perturbed by noble gas atoms the calculation of the absorption profile is possible only in a few specific thermodynamic conditions [10]. Therefore, one should resort to a semiclassical description of velocity-changing and speed-dependent effects, following the pioneering work of Rautian and Sobelman [11]. As a result, a variety of models are available to describe different physical situations, more or less successfully [9].

Hitherto, the complexity of sufficiently refined line-shape models has led to a major requirement for DBT, namely, the selection of a well-isolated line, in order to minimize the number of free parameters in the nonlinear fitting procedure. On the other hand, due to the statistical correlation between the Doppler width and collisional broadening and narrowing parameters, entering into play in the fitting process, relatively large fluctuations of the retrieved Doppler widths are typically encountered, ultimately limiting the achievable precision. This is the reason why high precision is pursued through acquisition of a very large number of spectra, up to several thousands for a precision level of $10 \mathrm{ppm}$ or better $[12,13]$.

Here, we propose a DBT approach in which the temperature is retrieved from a manifold of absorption lines, rather than from a single and well-isolated line. This is accomplished without sacrificing the complexity of the line profiles. Even with a relatively small number of spectra, the method provides strong elements for the selection of the most appropriate lineshape model and reduces consistently fluctuations resulting from statistical correlation.

\section{EXPERIMENTAL SETUP}

Three closely spaced $\mathrm{NH}_{3}$ transitions were probed, namely, the $\operatorname{sR}(6,2)$, $\operatorname{sR}(6,6)$, and $\operatorname{sR}(6,1)$ lines of the $\nu_{2}$ band, using a comb-calibrated absorption spectrometer based upon a continuous wave, room-temperature, distributed feedback 


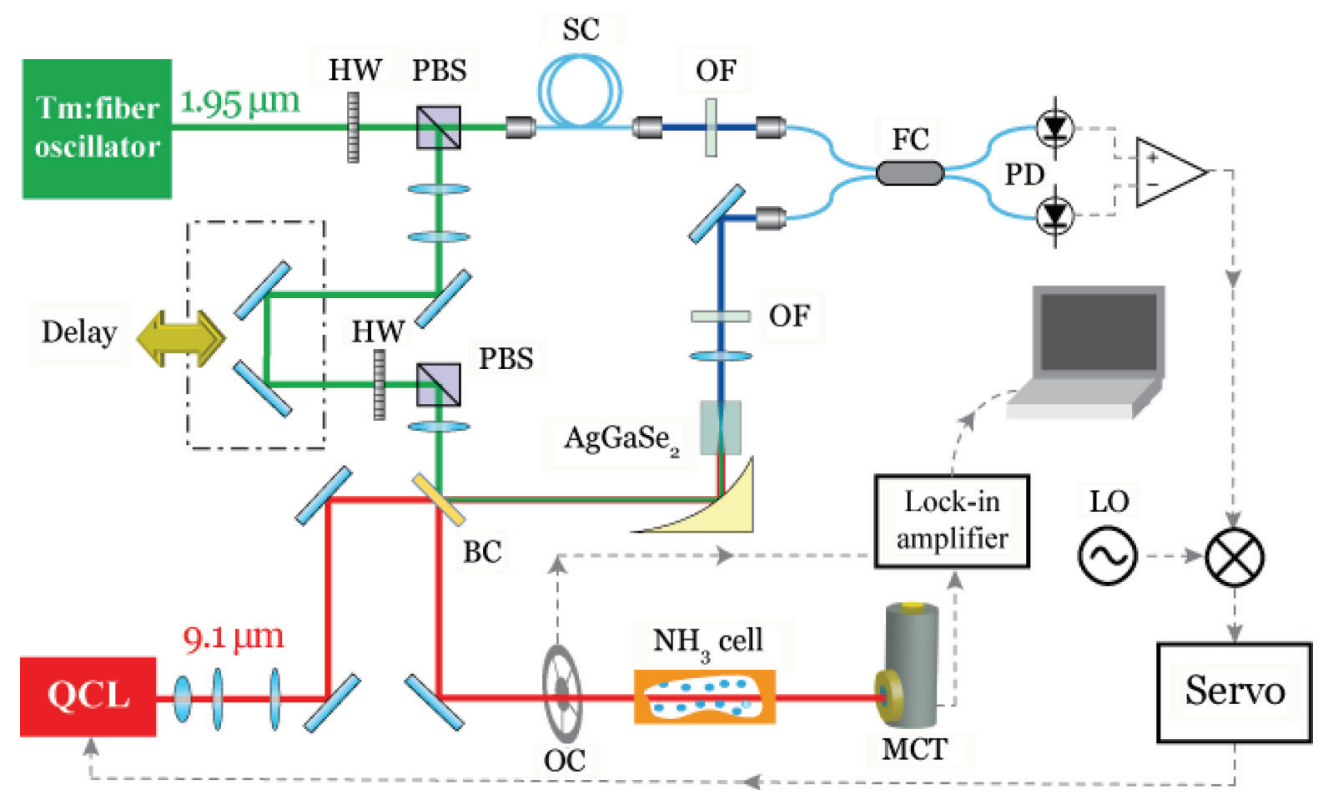

FIG. 1. (Color online) Experimental setup used for comb-assisted absorption spectroscopy of ammonia at 9.07 $\mu \mathrm{m}$. QCL, quantum cascade laser; PBS, polarizing beam splitter; SC, supercontinuum fiber; HW, half waveplate; OF, optical filter; FC, fiber coupler; PD, photodetector; $\mathrm{BC}$, beam combiner; LO, local oscillator at radio frequency; MCT, mercury-cadmium-telluride detector; OC, optical chopper.

quantum cascade laser (QCL) at $9.07 \mu \mathrm{m}$ referenced to a thulium-fiber femtosecond laser, as displayed in Fig. 1. The Tm:fiber comb delivers up to $2.2 \mathrm{~W}$ at $1.95 \mu \mathrm{m}$ with a repetition rate of about $85 \mathrm{MHz}$, and it is equipped with a piezoelectric stage and a locking servo allowing its repetition rate to be stabilized against a radio frequency (rf) synthesizer and then finely scanned by more than $2 \mathrm{kHz}$. Driven by a commercial current source with a current noise spectral density as small as $2 \mathrm{nA} / \mathrm{Hz}^{1 / 2}$, the QCL emits on a single mode up to $10 \mathrm{~mW}$. It is phase locked to the Tm:fiber frequency comb via a sum-frequency generation (SFG) scheme (see Ref. [8] for details). To this purpose, a 5-mm-long $\mathrm{AgGaSe}_{2}$ crystal has been selected for its high nonlinearity, low spatial walk-off, and broad transparency range. With powers of nearly $6 \mathrm{~mW}$ from the QCL and $100 \mathrm{~mW}$ from the Tm:fiber laser, the optical power of the SFG radiation is about $50 \mathrm{nW}$ at around $1.6 \mu \mathrm{m}$. The $1.6-\mu \mathrm{m}$ comb is fiber coupled and heterodyned with the supercontinuum (SC) radiation generated by focusing nearly $300 \mathrm{~mW}$ of the Tm:fiber laser into a $15-\mathrm{cm}$-long highly nonlinear fiber with zero dispersion wavelength close to $1.95 \mu \mathrm{m}$. The beat note signal is maximized by filtering the $\mathrm{SC}$ in order to match it with the 5-nm-wide spectrum of the SFG comb and by employing a double-balanced detection scheme. The beat note exhibits a signal-to-noise ratio of about $30 \mathrm{~dB}$ when acquired with a resolution bandwidth (RBW) of $300 \mathrm{kHz}$ and the QCL in free running conditions, as reported in Fig. 2. The phase lock between comb and QCL is achieved by comparing the beat note signal with a rf local oscillator and by feeding the error signal back to the QCL current. The two rf sources were phase locked to a GPS-disciplined $\mathrm{Rb}$ clock. A $100-\mathrm{kHz}$ servo bandwidth was found to be enough to shrink the 1-MHz-wide beat note below the instrumental resolution with a signal-to-noise ratio as high as $56 \mathrm{~dB} / \mathrm{Hz}$, as shown in the inset of Fig. 2. This feature indicates a remarkably low integrated phase noise of less than $1 \mathrm{rad}$. In such tight locking conditions, the QCL frequency could be precisely and repeatedly tuned over several $\mathrm{GHz}$ by varying the comb repetition rate. Moreover, the QCL emission linewidth could be reduced down to $25 \mathrm{kHz}$ at 1-ms observation time [8]. The $\mathrm{NH}_{3}$ absorption was probed by sending a small fraction of the QCL beam through a 11-cm-long cell, whose transmittance was synchronously monitored by a nitrogen-cooled MCT detector followed by a lock-in amplifier. Pressure and temperature of the cell were, respectively, measured by means of a capacitive gauge and a precision platinum resistance thermometer (Pt100), connected to a 6.5-digit volt meter. The Pt100 thermometer was calibrated at the triple point of water and at the gallium melting point with an overall accuracy of $\sim 0.01 \mathrm{~K}$. The correct scaling parameter between the comb repetition rate and the QCL frequency was

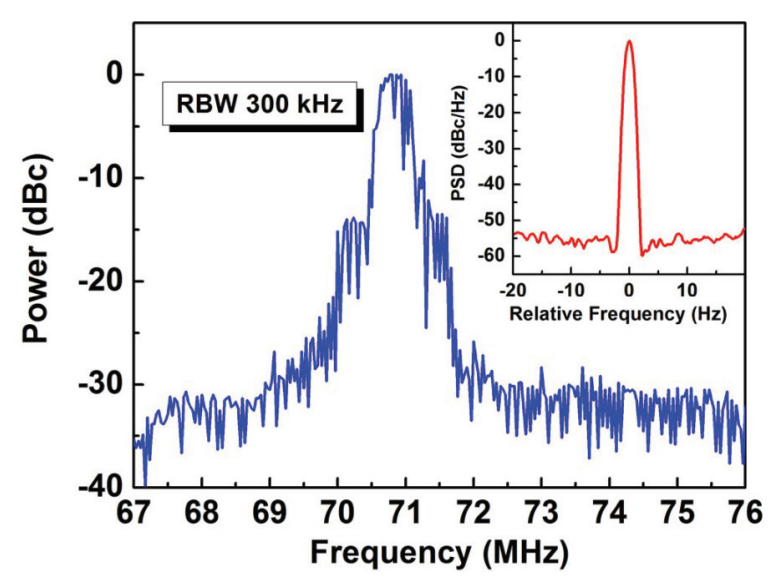

FIG. 2. (Color online) Beat-note signal between the Tm:fiber frequency comb and the QCL in free-running and phase-locking (in the inset) conditions. 
unambiguously found by using HITRAN data [14], whose uncertainty was below half of the repetition rate.

The gas cell was periodically evacuated and filled with pure $\mathrm{NH}_{3}$ samples, at a pressure between 40 and 300 mTorr. The experiment was performed at $\sim 296 \mathrm{~K}$, with a passive temperature stabilization of the gas cell. Repeated acquisitions were done at each pressure, leading to a total number of acquired spectra equal to 90 . Each laser scan was nearly $1 \mathrm{GHz}$ wide and consisted of $10^{4}$ frequency steps, which covered an overall time interval of about $16 \mathrm{~min}$.

\section{RESULTS AND DISCUSSION}

An example spectrum is shown in the upper panel of Fig. 3. Spectral analysis required a multiple-line, nonlinear, least-squares-fitting code, which was developed under the MATLAB environment. Being the absorption process ruled out by the well-known Beer-Lambert law, each spectrum could be fitted to the function below:

$$
\begin{aligned}
P(\tilde{v})= & \left(P_{0}+P_{1} \tilde{v}\right) \exp \left\{-\left[A_{1} F\left(\tilde{v}-\tilde{v}_{1}\right)+A_{2} F\left(\tilde{v}-\tilde{v}_{2}\right)\right.\right. \\
& \left.\left.+A_{3} F\left(\tilde{v}-\tilde{v}_{3}\right)\right]\right\},
\end{aligned}
$$

where $P(\tilde{v})$ is the transmitted power; $\tilde{v}$ is the absolute wave number (expressed in $\mathrm{cm}^{-1}$ ); $P_{0}$ and $P_{1}$ account for a linear variation of the incident power while tuning the laser frequency; and $\tilde{v}_{i}$ and $A_{i}$ are the line-center frequency and the integrated absorbance, respectively, for the ith line, the index $i$ ranging between 1 and 3 . The normalized shape function is indicated by the letter $F$ and is characterized by a set of parameters whose values are expected to vary from one line to the other. We tested and compared a series of semiclassical models of increasing complexity: the modified Voigt profile (MVP), the soft-collision profile of Galatry (GP), the hard-collision profile of Nelkin and Ghatak (NGP), and the symmetric version of the speed-dependent Voigt profile (SDVP). Their respective basic equations can be easily found elsewhere in the literature [15]. For each model, the Doppler

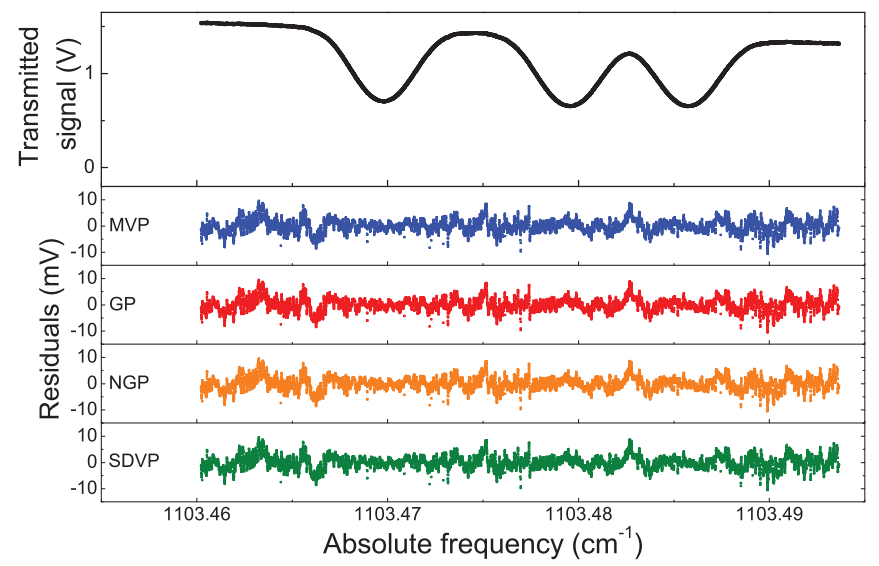

FIG. 3. (Color online) Experimental ammonia spectrum near $\lambda=9.07 \mu \mathrm{m}$, at a pressure of about 0.1 Torr and a temperature of $295.18 \mathrm{~K}$. Absolute residuals do not show any particular trend, thus demonstrating that the hypothesis of a linear variation for the laser power was very well satisfied. Their root-mean-square value (of about $2.4 \mathrm{mV}$ ) is consistent with a signal-to-noise ratio of about 650 , for an equivalent noise detection bandwidth of $2.5 \mathrm{~Hz}$. widths were constrained so as to obey the following scaling law:

$$
\frac{\Delta \tilde{v}_{D, i}}{\Delta \tilde{v}_{D, j}}=\frac{\tilde{v}_{i}}{\tilde{v}_{j}},
$$

independently of pressure, $i$ and $j$ referring to a pair of $\mathrm{NH}_{3}$ lines. This comes from the linear dependence of the Doppler width (HWHM) on the line-center frequency, according to the well-known formula

$$
\Delta \tilde{v}_{D}=\frac{\tilde{\nu}_{\text {line }}}{c} \sqrt{2 \ln 2 k_{\mathrm{B}} \frac{T}{M}},
$$

where $T$ is the thermodynamic temperature of the absorbing medium, $c$ is the speed of the light, $k_{\mathrm{B}}$ is the Boltzmann constant, $\tilde{v}_{\text {line }}$ is the line-center frequency, and $M$ is the mass of the molecule. The use of Eq. (2) as a constraint in the multiple-spectrum fitting procedure is expected to remove at least partially the correlation issues, thus reducing significantly the statistical uncertainty on the spectroscopic determination of $T$.

The lower panels of Fig. 3 clearly demonstrate that all the tested models were successful in reproducing the experimental spectrum, within the noise. This peculiarity emerges in the analysis of all recorded spectra. However, because of the approximate nature of the models, a successful fit does not necessarily mean that the retrieved parameters are physically meaningful.

For the MVP model, free parameters were $T, P_{0}, P_{1}, \tilde{v}_{i}, A_{i}$, and $\Gamma_{i}$ (with $i$ varying between 1 and 3), this latter quantity representing the homogeneous width (HWHM) of the ith line. For profiles with increasing complexity, other quantities were treated as free parameters: the effective frequency of the velocity changing collisions $(\beta)$ for Galatry fits and the total collision frequency $(\Omega)$ for the NGP model. In the fitting procedure, the frequencies $\beta$ and $\Omega$ were forced to be equal for all three spectral lines. As for the SDVP model, we recall that, following the Berman-Pickett approach, a hypergeometric dependence of the collisional width $(\Gamma)$ on the absorber speed $\left(v_{a}\right)$ was considered according to the equation below [16]:

$$
\Gamma\left(v_{a}\right)=\frac{\Gamma_{0}}{(1+\lambda)^{m / 2}} \cdot M\left[-\frac{m}{2} ; \frac{3}{2} ;-\lambda\left(\frac{v_{a}}{v_{a_{0}}}\right)^{2}\right],
$$

where $\Gamma_{0}=<\Gamma\left(v_{a}\right)>$ is the average collisional width over molecular speeds, $\lambda=m_{p} / m_{a}$ is the mass ratio of perturbing and absorbing molecules (equal to 1 in our experiment), $v_{a 0}$ is the most probable speed of the absorbing molecules, and $M[a ; b ; x]$ is the confluent hypergeometric function. The quantity $m$ is given by the ratio $(q-3) /(q-1)$, where $q$ is the exponent determining the collision interaction potential, which is assumed of the form $V(R) \propto R^{-q}, R$ being the intermolecular distance [17]. In the present work, we set $q=4$ for each of the three lines, while treating $\Gamma_{0}$ as a free parameter.

The choice of the line-shape model becomes relevant when moving to the spectroscopic determination of the gas temperature. For each spectrum, after the application of the nonlinear least-squares-fitting procedure, we calculated the ratio between the retrieved temperature $\left(T_{S}\right)$ and that of the pt100 thermometer $\left(T_{\mathrm{pt100}}\right)$. In Fig. 4 , these ratios are plotted as a function of the integrated absorbance (which is in turn proportional to the molecular number density, $N$ ). 


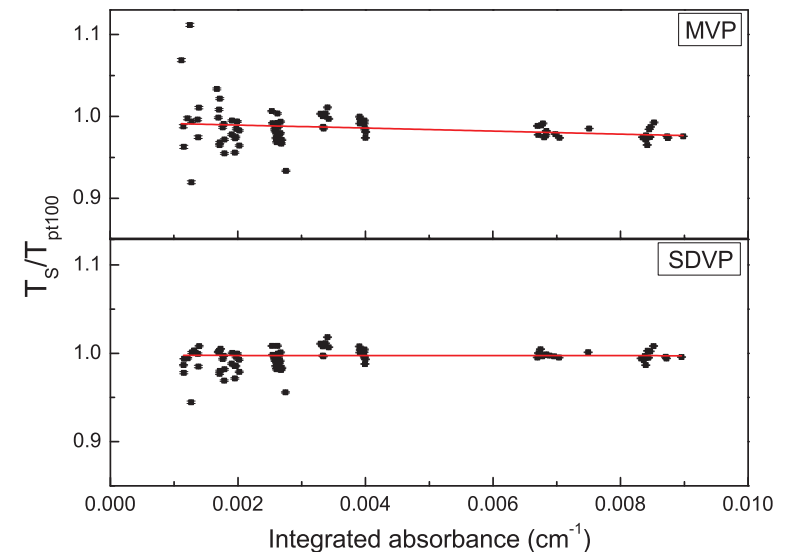

FIG. 4. (Color online) Comparison between the spectroscopic determinations of the gas temperature, as retrieved by means of the MVP and SDVP models, and the values measured by means of the pt100 thermometer. Best-fit lines (in red) are also shown.

In particular, Fig. 4 shows the results obtained from the application of the MVP and SDVP models, respectively reported in the upper and lower plots. If the selected model is appropriate for a physical description of the shape in the whole pressure range, the temperature ratio should be independent of $N$ and equal to 1 , within the experimental uncertainty. A weighted linear fit is performed in order to retrieve the slope of a possible linear dependence on $N$. MVP data clearly exhibit a trend with a negative slope, while SDVP data provide a zero slope. This means that the Voigt profile, even if the Doppler width is treated as a free parameter, is not sufficient for a physical description of the observed shapes, while the speed-dependent Voigt model does much better. Moreover, the zero slope of the SDVP data confirms the validity of our choice for the $q$ value, which is very close to that $(q=4.125)$ reported in a recent study on speed-dependent effects in self-colliding $\mathrm{NH}_{3}$ molecules [18]. It is worth noting that, at the present noise level, no line asymmetry could be evidenced, this being the reason why we did not consider the speed dependence of the pressure shift. SDVP fits were also repeated treating the three Doppler widths as independent (free) parameters. The analysis of the covariance matrix shows that the statistical correlation between Doppler and collisional widths reduces from -0.96 to -0.80 when moving from the unconstrained case to the constrained one. Such a reduction represents an important outcome for the aims of DBT, and it can be even more pronounced when a larger number of lines are involved.

Apart from SDVP, the only model capable of providing zero slope within one standard deviation was the Galatry profile, as shown in Fig. 5. Both MVP and NGP provide negative slopes, as expected when narrowing effects are not properly accounted for. However, for the ratios resulting from Galatry fits, the slope exhibits the largest uncertainty. This attests the difficulties of the Galatry model in reproducing the observed narrowing effects, which can be mostly ascribed to speed dependence rather than to Dicke narrowing. This statement is further supported by the negative value of the slope emerging from the application of the Nelkin-Ghatak model. For the speed-dependent Voigt profile, we also considered the quadratic approximation (SDVP-Q), resulting from the first-

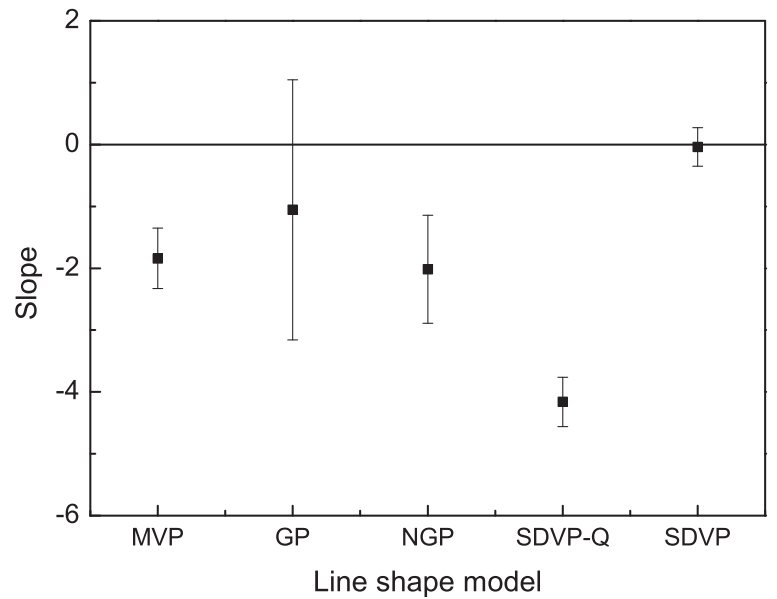

FIG. 5. Slope of the best-fit lines obtained for different temperature-ratio data resulting from the application of different line-shape models.

order expansion of the hypergeometric dependence of Eq. (4) [19]. Figure 5 clearly shows the failure of this approximation.

The weighted mean of the SDVP temperature ratios gives 0.99772 (5), which differs from 1 by $2.27 \times 10^{-3}$. Its statistical uncertainty indicates that a precision better than $5 \times 10^{-5}$ can be achieved in the spectroscopic determination of the gas temperature, as a result of the analysis of a restricted number of spectra, of the order of 100 . Possible sources of systematical deviations will be the subject of a future investigation, once a significant improvement of the temperature stability and homogeneity of the gas cell is made by adopting an isothermal cell similar to that described in Ref. [20]. In those conditions, more sophisticated line-shape models will be tested, like the speed-dependent Galatry or the speed-dependent NelkinGhatak profiles [15].

\section{CONCLUSIONS}

In conclusion, we have demonstrated that comb-assisted QCL-based infrared absorption spectroscopy is a powerful DBT method. From the experimental point of view, the use of a Tm:fiber laser frequency comb ensured a highly accurate, absolute, and repeatable frequency axis underneath each absorption spectrum, along with an efficient reduction of the QCL emission width. Our approach, involving three spectral lines of a single molecular species, rather than a well-isolated line, turns out to be reliable and highly precise, provided that a proper choice of the line-shape model is made. Our precision, if properly scaled to simulate the situation of a much larger number of spectra, is already better than the best result obtained so far [13]. If applied to a system under tightly controlled thermodynamic conditions, our DBT approach is likely to open new interesting perspectives for a spectroscopic determination of the Boltzmann constant with an uncertainty at the ppm level.

\section{ACKNOWLEDGMENTS}

D.G., M.M., and L.G. warmly thank IMRA America for kind hospitality. 
[1] C. Daussy, S. Briaudeau, M. Guinet, A. Amy-Klein, Y. Hermier, C. J. Bordé, and C. Chardonnet, in Laser Spectroscopy, edited by E. A. Hinds, A. Ferguson, and E. Riis (World Scientific, Singapore, 2005), pp. 104-111.

[2] C. Daussy, M. Guinet, A. Amy-Klein, K. Djerroud, Y. Hermier, S. Briaudeau, C. J. Bordé, and C. Chardonnet, Phys. Rev. Lett. 98, 250801 (2007).

[3] G. Casa, A. Castrillo, G. Galzerano, R. Wehr, A. Merlone, D. Di Serafino, P. Laporta, and L. Gianfrani, Phys. Rev. Lett. 100, 200801 (2008).

[4] K. Djerroud, C. Lemarchand, A. Gauguet, C. Daussy, S. Briaudeau, B. Darquié, O. Lopez, A. Amy-Klein, C. Chardonnet, and C. J. Bordé, C. R. Phys. 10, 883 (2009).

[5] A. Castrillo, G. Casa, A. Merlone, G. Galzerano, P. Laporta, and L. Gianfrani, C. R. Phys. 10, 894 (2009).

[6] A. Castrillo, E. Fasci, G. Galzerano, G. Casa, P. Laporta, and L. Gianfrani, Opt. Express 18, 21851 (2010).

[7] K. M. Yamada, A. Onae, F.-L. Hong, H. Inaba, and T. Shimizu, C. R. Phys. 10, 907 (2009).

[8] A. A. Mills, D. Gatti, J. Jiang, C. Mohr, W. Mefford, L. Gianfrani, M. Fermann, I. Hartl, and M. Marangoni, Opt. Lett. 37, 4083 (2012).

[9] J. M. Hartmann, C. Boulet, and D. Robert, Collisional Effects on Molecular Spectra. Laboratory Experiments and
Models, Consequences for Applications (Elsevier, New York, 2008).

[10] R. Wehr, R. Ciurylo, A. Vitcu, F. Thibault, J. Drummond, and A. May, J. Mol. Spectrosc. 235, 54 (2006).

[11] S. Rautian and I. Sobelman, Sov. Phys. Uspekhi-USSR 9, 701 (1967).

[12] C. Lemarchand, K. Djerroud, B. Darquié, O. Lopez, A. AmyKlein, C. Chardonnet, C. Bordé, S. Briaudeau, and C. Daussy, Int. J. Thermophys. 31, 1347 (2010).

[13] C. Lemarchand, M. Triki, B. Darquié, C. J. Bordé, C. Chardonnet, and C. Daussy, New J. Phys. 13, 073028 (2011).

[14] L. Rothman et al., J. Quantum Spectrosc. Radiat. Transfer 110, 533 (2009).

[15] R. Ciuryło, Phys. Rev. A 58, 1029 (1998).

[16] R. Ciurylo and J. Szudy, J. Quantum Spectrosc. Radiat. Transfer 57, 411 (1997).

[17] H. M. Pickett, J. Chem. Phys. 73, 6090 (1980).

[18] M. Triki, C. Lemarchand, B. Darquié, P. L. T. Sow, V. Roncin, C. Chardonnet, and C. Daussy, Phys. Rev. A 85, 062510 (2012).

[19] F. Rohart, H. Mäder, and H.-W. Nicolaisen, J. Chem. Phys. 101, 6475 (1994).

[20] A. Merlone, F. Moro, A. Castrillo, and L. Gianfrani, Int. J. Thermophys. 31, 1360 (2010). 